\title{
El análisis espacial del uso del suelo en la periferia del Gran Resistencia y Gran Corrientes, mediante la aplicación de geoindicadores
}

JORGE A. ALBERTO, ANÍBAL M. MIGNONE, GUILLERMO A. ARCE, SILVINA LOPEZ

Centro de Geociencias Aplicadas, Universidad Nacional del Nordeste (UNNE), Argentina jaalberto@hotmail.com, animarmig@hotmail.com, gar_arce@yahoo.com.ar, silvina_ lopez@hotmail.com

\section{RESUMEN}

Esta investigación analiza la dinámica y evolución del uso del suelo en la periferia de los aglomerados Gran Resistencia y Gran Corrientes en el siglo XXI. Para ello, se considera la aplicación de geoindicadores que, desde la concepción geográfica y sistémica, permiten proporcionar herramientas para comprender las raíces históricas, la situación actual y la evolución físico-social de las ciudades, a fin de reconocer la tendencia y dinámica del uso del suelo, además de proyectar y evaluar los aspectos socio-ambientales. El marco de análisis dominante se basa en el principio de causalidad (marco ImpulsoEstado- Respuesta), ya que se considera que las actividades humanas ejercen presión sobre el ambiente y cambian las características cualitativas y cuantitativas de los recursos. Se trabaja con fuentes bibliográficas, cartográficas y estadísticas que permiten reconocer los fenómenos antes mencionados.

PALABRAS CLAVE: geoindicadores; Gran Resistencia; Gran Corrientes; expansión urbana; uso suelo; periurbano. 


\section{ABSTRACT}

\section{SPATIAL ANALYSIS OF LAND USE ON THE PERIPHERY OF THE GRAN RESISTENCIA AND GRAN CORRIENTES, APPLYING GEOINDICATORS}

This research analyses the dynamics and evolution of land use in the periphery of the Gran Resistencia and Gran Corrientes agglomerates in the 2Ist century. For this, the application of geoindicators is considered, which from the geographical and systemic conception, allows to provide tools to understand the historical roots, the current situation and the physical-social evolution of cities, in order to recognize the trend and dynamics of land use, besides projecting and evaluating the socio-environmental aspects. The dominant analysis framework is based on the principle of causality (Impulse - State - Response framework), since it is considered that human activities exert pressure on the environment and change the qualitative and quantitative characteristics of resources. We work with bibliographic, cartographic and statistical sources that allow us to recognize the aforementioned phenomes.

KEYWORDS: geoindicators; Gran Resistencia; Gran Corrientes; urban expansion; land use; peri-urban. 


\section{RESUMO}

\section{ANÁLISE ESPAÇIAL DO USO DO SOLO NA PERIFERIA DE GRAN RESISTENCIA E GRAN CORRENTES, ATRAVÉS DA APLICAÇÃO DE GEOINDICADORES}

Esta pesquisa analisa a dinâmica e evoluçáo do uso da terra na periferia dos aglomerados Gran Resistencia e Gran Corrientes no século XXI. Para isso, considera-se a aplicação de geoindicadores, que a partir da concepção geográfica e sistêmica, permite fornecer ferramentas para compreender as raízes históricas, a situação atual e a evolução físico-social das cidades, a fim de reconhecer a tendência e dinâmica do uso da terra. , além de projetar e avaliar os aspectos socioambientais. A estrutura de análise dominante é baseada no princípio da causalidade (estrutura Impulso - Estado - Resposta), uma vez que se considera que as atividades humanas exercem pressão sobre $\mathrm{o}$ ambiente e modificam as características qualitativas e quantitativas dos recursos. Trabalhamos com fontes bibliográficas, cartográficas e estatísticas que nos permitem reconhecer os fenômenos supracitados.

PALAVRAS-ChaVE: geoindicadores; Gran Resistencia; Gran Corrientes; expansão urbana; uso do solo; peri-urbano. 


\section{Introducción}

En la Argentina, como en los restantes países de América Latina, la mayoría de las ciudades experimenta un proceso de expansión periférica, con una ocupación discontinua del espacio y con un avance creciente sobre áreas periurbanas y rurales. El periurbano constituye un "territorio de borde" que está sometido a procesos económicos relacionados con la valorización capitalista del espacio, como consecuencia de la incorporación de nuevas tierras a la ciudad. La ocupación del suelo generalmente se realiza de una manera no planificada, constituyendo un espacio de gran heterogeneidad, donde pueden registrarse fuertes problemáticas sociales y ambientales.

En la actualidad, los aglomerados del Gran Resistencia y Gran Corrientes muestran una expansión urbana hacia las periferias, apoyados por diferentes factores que impulsan fenómenos relacionados con:

- la puesta en valor de los espacios intersticiales mediante proyectos de inversiones públicas y privadas,

- las intervenciones sobre sectores que aún no han adquirido consistencia urbana,

- las políticas de Estado o la dinámica inmobiliaria,

- la construcción de nuevas vías de comunicación.

Todo ello, se produce sobre un medio físico que establece limitaciones al avance de las ciudades, pero no ha impedido las nuevas modalidades en el uso del suelo y en la configuración periférica de ambas ciudades.

En el marco del contexto mencionado anteriormente, esta investigación analiza la dinámica y evolución del uso del suelo en la periferia de los aglomerados Gran Resistencia y Gran Corrientes en el siglo XXI, considerando el uso de los geoindicadores, a fin reconocer la tendencia y dinámica del uso del suelo, además de proyectar y evaluar los aspectos socio-ambientales de los ejidos urbano mencionados.

Los geoindicadores, pueden ser aplicados tanto en fenómenos específicos con comportamientos cíclicos sobre la superficie terrestre en largos periodos de tiempo para determinar cambios en el paisaje, como en espacios con dinámicas complejas en su evolución en cortos periodos de tiempo, como es el caso de la dinámica del crecimiento urbano y su incidencia sobre entornos rurales (Alberto, 2012). 
Este trabajo se enmarca en un proyecto mayor, que se desarrolla en el Centro de Geociencias Aplicadas (Humanidades-Ingeniería, UNNE), referido a la aplicación de geoindicadores a los distintos aspectos de la problemática ambiental del territorio, y que toma como objeto de estudio a los centros urbanos del Gran Resistencia y el Gran Corrientes y los ámbitos periurbanos próximos.

Para cumplir los objetivos, se analizaron fuentes de información estadística y cartográfica obtenidas de los organismos nacionales (base de datos censales del Instituto Nacional de Estadística y Censos, INDEC), provinciales (Direcciones de Catastro de las provincias de Chaco y Corrientes) y municipales (Direcciones de Catastro de los municipios). Asimismo, se trabajó con imágenes satelitales obtenidas del Google Earth y se realizó la tarea de campo efectuando recorridos por la periferia de ambos aglomerados.

\section{Enfoque Metodológico}

La International Union of Geological Sciences (IUGS) decidió en I992, establecer indicadores (magnitudes, frecuencias, ritmos y tendencias) de procesos y fenómenos que ocurren en la superficie terrestre y están sujetos a cambios ambientales que son significativos para utilizarlos en los informes referidos al uso del suelo y al manejo de los ecosistemas (IUGS, 20I2). De esta manera, en el marco de una concepción geográfica y sistémica, los geoindicadores deben proporcionar herramientas que permitan la definición de escenarios, fundamentados en el conocimiento científico y en la capacidad de análisis y proyección del investigador. En el primero, para comprender las raíces históricas, la situación actual y la evolución físico-social y en la segunda para proyectar y evaluar los aspectos socio- ambientales.

Los geoindicadores, pueden ser aplicados tanto en espacios con dinámicas complejas en su evolución en cortos períodos de tiempo (por ej. dinámica y evolución del crecimiento urbano y su incidencia sobre entornos rurales) o en fenómenos específicos con comportamientos cíclicos sobre la superficie terrestre en largos períodos de tiempo (por ej. fenómenos de inundaciones y sequias y su incidencias sobre las actividades sociales) para determinar cambios importantes en el paisaje (Alberto, 20I2), en forma tal que puedan ser difundidas, entre plani- 
ficadores y personas que toman decisiones (Gupta, 2000) o bien, en la toma de conciencia a través de la educación formal e informal por medio de actividades sencillas e incluidas en la currícula educativa de los proyectos en instituciones educativas de nivel medio (Bataglia, 20I2). Es decir, pueden ayudar a determinar impactos y problemáticas ambientales derivadas sobre el medio estudiado, monitorear ecosistemas de forma continua, cambios en el uso del suelo, seleccionar prácticas de manejo y determinar condiciones de base previas a todo proyecto de desarrollo urbano complementado con servicios e infraestructuras derivadas (Coltrinari, 200I).
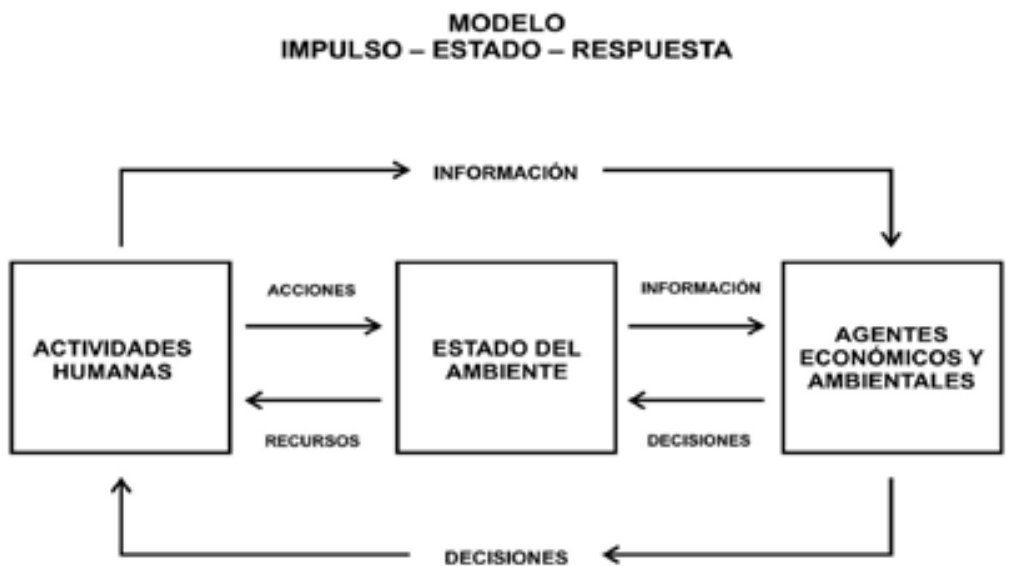

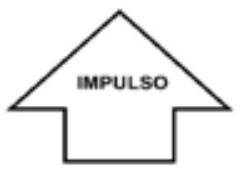

Indicadores de IMPULSO

externalidades directas de las actividades humanas, y tendencias o caracteristicas de las actividades que crean extemalidades ambiontales

Figura 1. Esquema del enfoque metodológico desarrollado por la Organización para la Cooperación y el Desarrollo

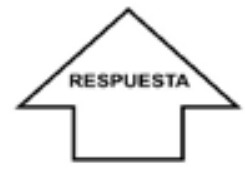

Indicadores de RESPUESTA

esfuerzos realizados por la sociedad o por el Estado para reducir o mitigar la degradación del ambiente.

Fuente: OCDE (1993)

Económicos (OCDE) 
El proceso de ocupación del espacio se analiza bajo el concepto de "marco causal", que se basa en el principio de causalidad (PresiónEstado-Respuesta), es decir que las actividades humanas ejercen presión sobre el ambiente y cambian las características cualitativas y cuantitativas de los recursos. Este marco conceptual, muy utilizado debido a su simpleza y a la posibilidad de aplicación a diferentes niveles y escalas, fue desarrollado por la Organización para la Cooperación y el Desarrollo Económicos (OCDE, 1993) a partir del modelo original de Presión-Respuesta de Friends y Raport (199I). Posteriormente las Naciones Unidas reemplazó el término "presión” por "impulso", para extender la dimensión ecológica a los indicadores sociales, económicos e institucionales (figura $\mathrm{I}$ ).

Por otra parte, los indicadores se entienden como variables que vuelven visibles o perceptibles fenómenos de interés, y miden y comunican información relevante; deben representar atributos considerados importantes por los tomadores de decisión y/o la población, para aportar a los procesos de toma de decisiones sobre el territorio (Gallopín, 2006).

El análisis de este trabajo se centra en el espacio periurbano, un área de gran heterogeneidad y crecimiento acelerado, donde pueden registrarse problemáticas sociales y ambientales agudas, un mercado del suelo poco transparente y proximidades conflictivas (Gutiérrez Puebla, 1984: 5). Este territorio de borde está sometido a procesos económicos relacionados con la valorización capitalista del espacio, como consecuencia de la incorporación real o potencial de tierras a la ciudad; de esta forma, en pocas partes de la superficie terrestre existen espacios que tengan tal diversidad y mezcla de usos del suelo, y donde el medio natural esté sometido a tan intensas presiones. (Capel, 1994: 139-I40 citados por López et al., 2015).

\section{Aspectos a tener en cuenta en el análisis de los espacios periurbanos}

Desde la concepción de "espacios periurbanos", la propuesta parte del tratamiento de la relación sociedad/naturaleza materializada en el proceso de ocupación de las áreas periféricas de una ciudad. El esquema teórico-metodológico de aproximación a la problemática (figura 2), implica tres indicadores de análisis espacial: 
a) La revalorización del enfoque espacio-temporal (indicador de estado), considerando el tiempo no como un continuo sino diferenciable en secuencias o periodos con rasgos específicos dados por interrelaciones particulares de los distintos hechos sociales, y materializado en espacio. Así, una perspectiva espacio-temporal permite comprender los mecanismos de la transformación del todo social y su expresión espacial.

b) La definición de las áreas sujetas a urbanización (indicador de impulso), se evaluó oportuno recurrir a las condiciones del sitio, entendidas no como algo dado y estático sino como componentes dinámicos del medio. Por ello, no solo se han incluido en esta categoría aquellas condiciones naturales originarias, sino también otras socialmente producidas que vienen a formar parte del medio tanto como las primeras. Particularmente, se destaca el caso de la topografía artificial creada a través de sucesivas nivelaciones del terreno, pues esta intervención condiciona las relaciones sociales a la vez que pasa a incorporarse y ser parte de ellas.

En función de la problemática que se aborda solo resultaron relevantes aquellas condiciones de sitio que se vinculan con el riesgo natural de inundación, es decir, geomorfología, topografía (cotas), pendiente, escurrimiento y permeabilidad del suelo.

c) El análisis de los agentes sociales (indicador de respuesta) que participan en este proceso, sus modalidades de gestión y también los patrones de valorización del espacio que sostienen, en tanto dichos agentes sociales, en su accionar, desarrollan sistemas de relaciones que se incorporan a la conformación espacial. 


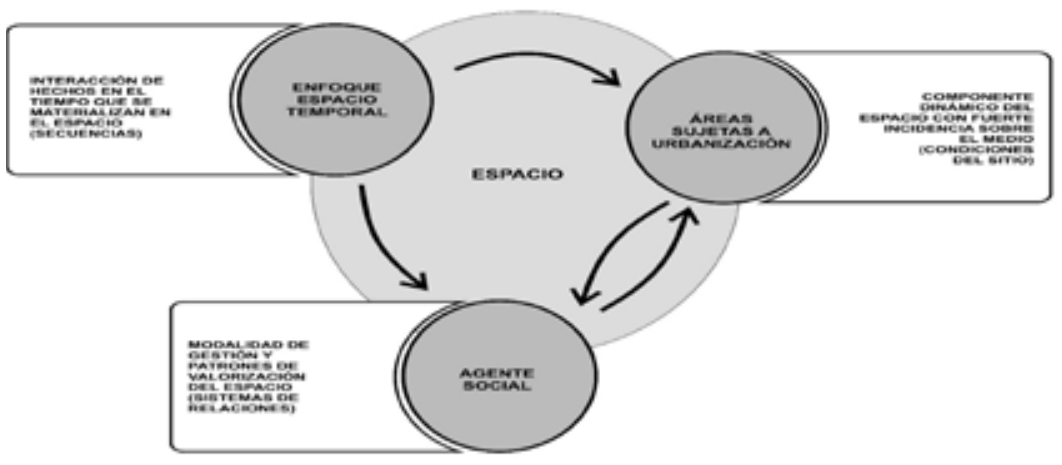

Fuente: elaboración propia.
Figura 2. Esquema teórico-metodológico de aproximación a la problemática

\section{El sitio: Morfología del Valle del río Paraná}

En el nordeste argentino se distinguen dos grandes unidades geográficas, el Chaco y la Mesopotamia (Orfeo, 1996) en cuya zona de contacto, recorrida por el Río Paraná, se localiza el núcleo bipolar regional ResistenciaCorrientes (Vapñarsky y Gorojovsky, 1990). Aproximadamente $30 \mathrm{Km}$ aguas arribas, al norte de estas ciudades, se produce el encuentro de los ríos Paraná y Paraguay en un ángulo casi recto producido, probablemente, por algún condicionamiento estructural (Popolizio, 1966) que da lugar a un cambio significativo en la dirección del escurrimiento. Sus caudales y carga sedimentaria tienen un comportamiento diferente (Orfeo, 1997) ya que el Paraná transporta menor carga sedimentaria en suspensión, siendo su grado de turbidez menor que el correspondiente a las aguas del Paraguay (Bonetto y Orfeo, 1984). Debido a la diferente turbidez de sus aguas (figura 3), estas no se mezclan sino que corren paralelas, separadas por conjuntos de islas y juntas cada tanto. Como consecuencia de este fenómeno existen dos canales: el del Paraná y el del Paraguay dando lugar a que la línea de vaguada, junto con la dinámica de su cauce, cambie con el tiempo (Popolizio, 1975, 1966; Bonetto y Orfeo, 1984)

En el encuentro de ambos ríos existe un significativo aumento de profundidad y otros fenómenos de sedimentación (Orfeo, 1997) que 
quedan reflejados sobre la margen derecha con un relieve positivo conocido como Cerrito Paraguayo que da el nombre a la gran isla que se extiende hacia el sur en territorio chaqueño, Isla del Cerrito, cubierta durante las grandes inundaciones.

En este sector, el valle mayor se amplía considerablemente en tanto que el curso principal tiende a apoyarse sobre la margen correntina, originando barrancas elevadas, terrazas fluviales y sectores de acumulación. Después de la confluencia del río Paraná con el río Paraguay el curso cambia de dirección hacia el SW formando dos canales: el del Paraná a la izquierda y del Paraguay a la derecha. El primero se apoya sobre la margen izquierda hasta donde está emplazada la ciudad de Corrientes (figura 4 y figura 5). En las barrancas, los sedimentos cuaternarios se sobreponen a los sedimentos del Mesopotamiense inferior, coronados por el "Asperón Guaranítico" (Formación Ituzaingó) y cubiertos por sedimentos cuaternarios que, junto con los fenómenos de erosión y empinamiento del talud sumergido, originan deslizamientos rotacionales y/o procesos de volcamiento (Popolizio, 1966). 
Nota: En la

imagen se puede observar que el río Paraná (color oscuro) transporta menor carga en suspensión de sedimentos con respecto al río Paraguay (color claro) lo que genera una diferencia de turbidez de sus aguas, las cuales no se mezclan, sino que corren paralelas.

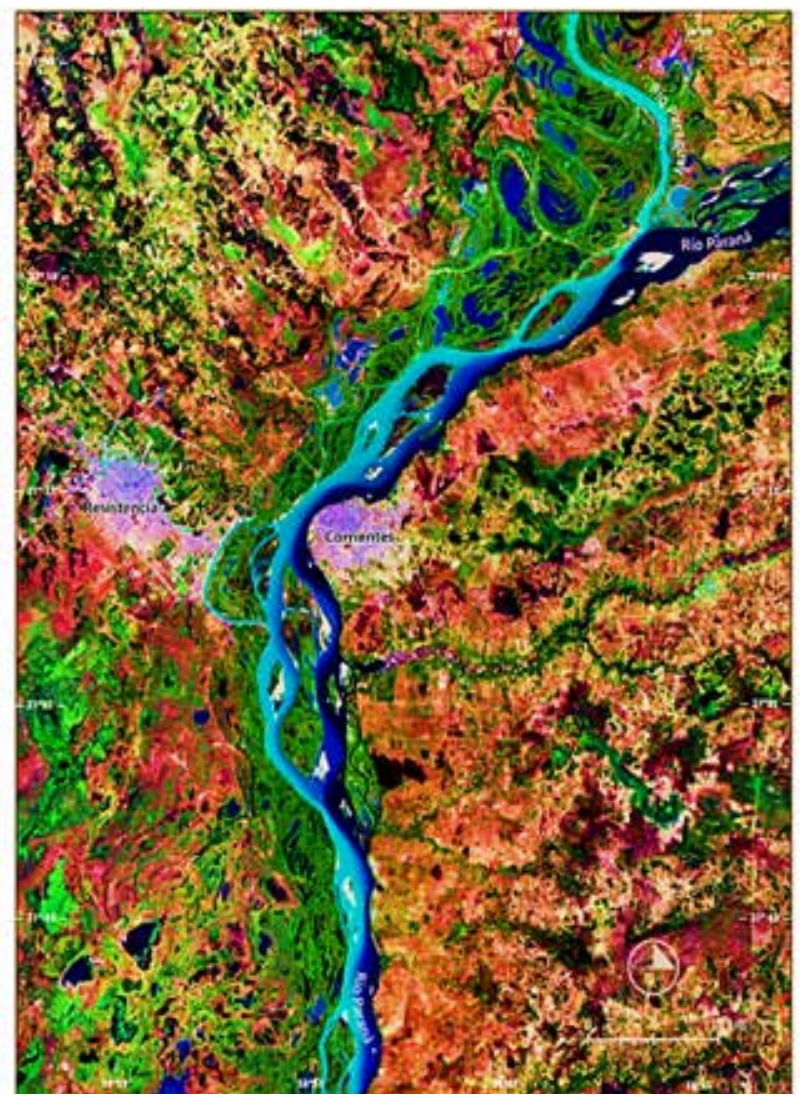

Figura 3. Encuentro del Río Paraná y el Río Paraguay

Fuente: Imagen LANDSAT_7_ETMXS_20020827_226_079_L2/ Fecha: 27-08-2002. Facilitada por el Centro de Geociencias Aplicadas, UNNE. 


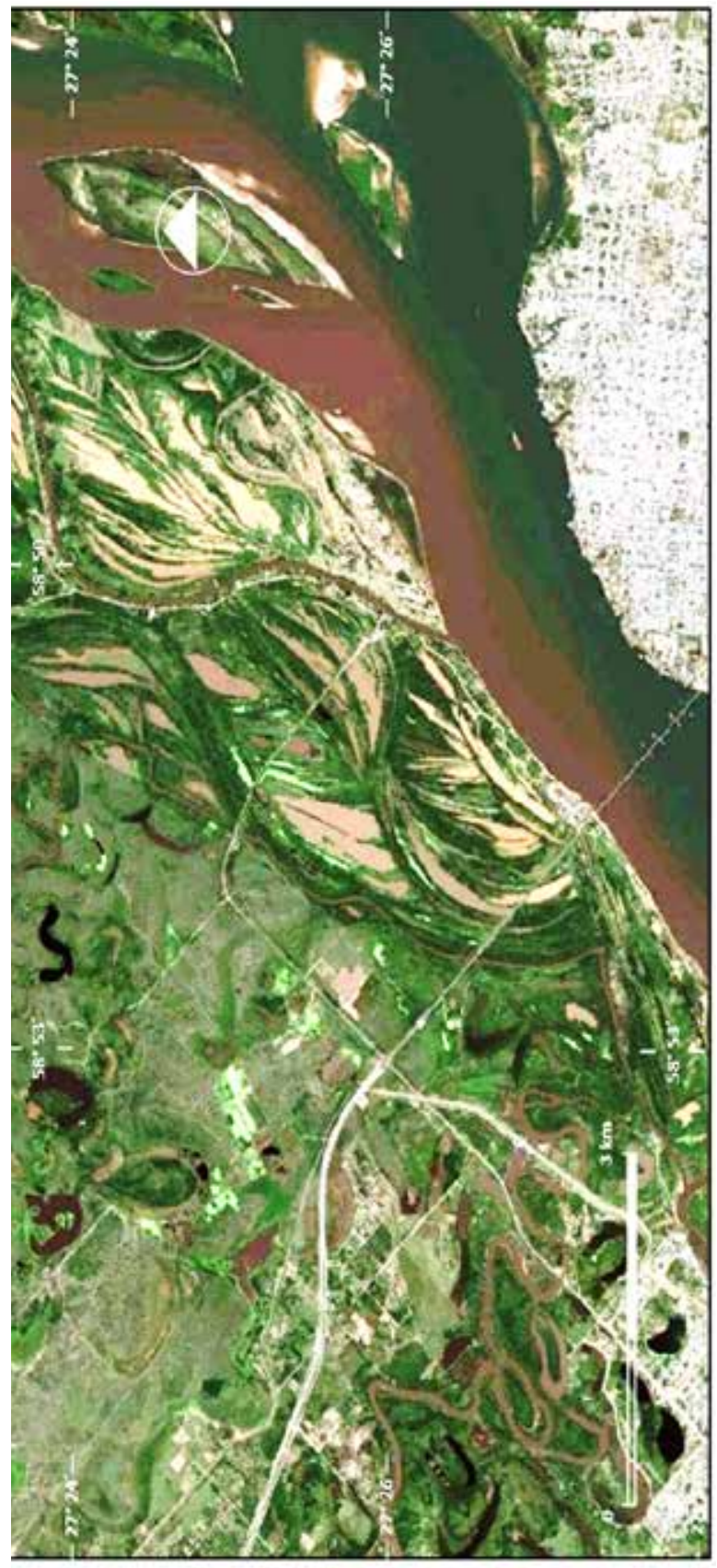

Figura 4. Ribera del Río Paraná
Fuente: Google

Earth, imagen

octubre 2010

Nota: En la imagen se pueden observar las formas fluviales que resultan del proceso de sedimentación, como el caso de la morfología isleña y espiras meándricas, que se han soldado a la ribera presentando una suave pendiente pero mucha irregularidad geomorfológica por diferentes fenómenos de diques marginales (Backswamp) sobre los que se desarrollan bosques riparios. 
Fuente: Popolizio (2001)

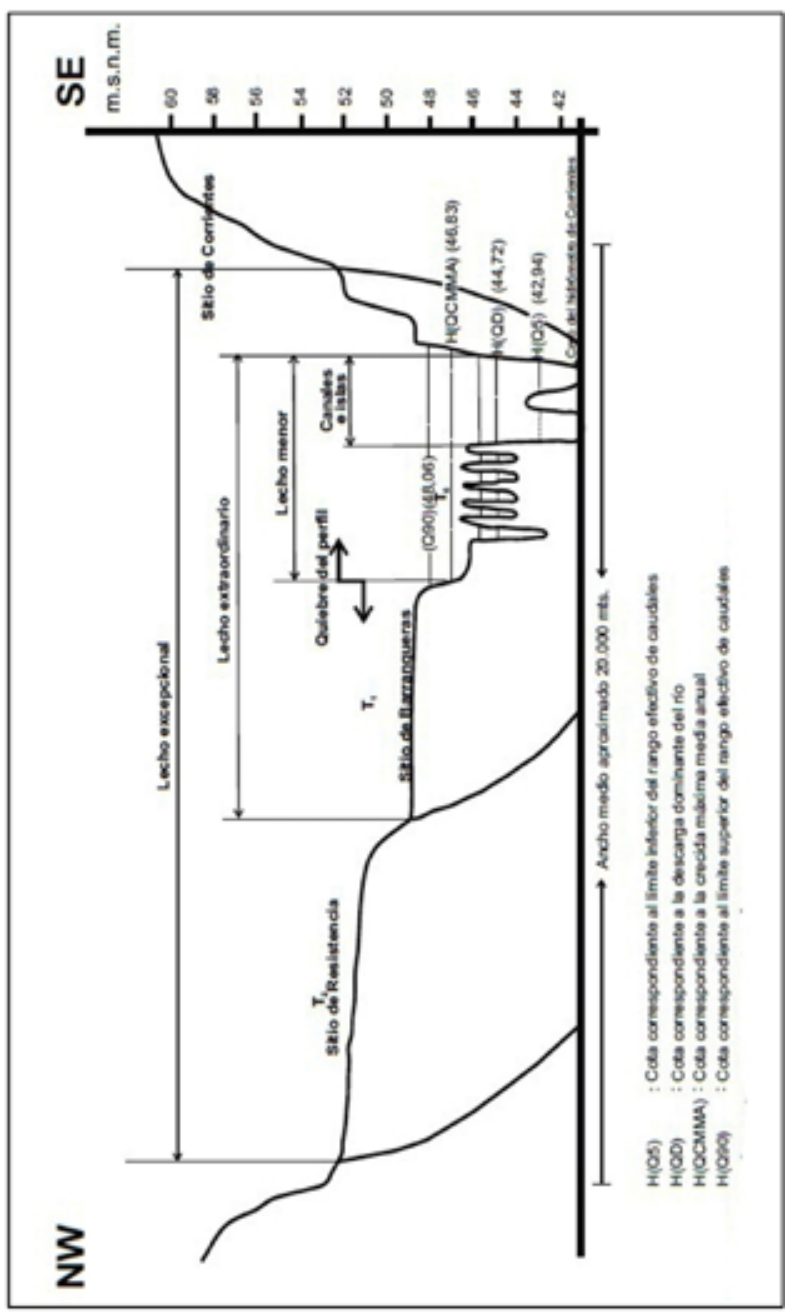

Figura 5. Perfil transversal exagerado y desfasado en la escala vertical del valle del río Paraná

Sobre la margen derecha del "canal" la tendencia general es a sedimentar, lo que ha dado lugar a profundas modificaciones en la morfología de la terraza $T_{\text {. }}$. A ellas se suman transformaciones que sucedieron y aún continúan desarrollándose en las formas fluviales como el caso de la morfología isleña y espiras meándricas que se han soldado a la 
ribera. Estas formas presentan suave pendiente pero mucha irregularidad geomorfológica por diferentes fenómenos de diques marginales (Backswamp) sobre los que se desarrollan bosques riparios (Popolizio, 200I; Neiff, Poi de Neiff y Casco, 2005). El proceso de sedimentación sobre la margen derecha ha dado lugar, en los últimos tiempos, a la sedimentación en la boca norte del riacho Barranqueras, que tiende a cerrarse impidiendo el ingreso al puerto del mismo nombre, lo cual obliga a continuas tareas de dragado.

\section{Morfología del sitio urbano del Gran Resistencia}

El sitio del Gran Resistencia y zonas aledañas presenta una constitución compleja en su morfología y génesis de sus sedimentos, ya que se superponen aluviones depositados por los ríos Paraná, Paraguay y Bermejo (Orfeo, 1996; Popolizio, 2000). El diseño que se destaca es el que corresponde a uno de los tantos valles abandonados del río Bermejo usados y reelaborados actualmente por los ríos Negro y Tragadero. La superposición de material sedimentario determinó que el área correspondiente al sitio de emplazamiento del complejo urbano del Gran Resistencia y localidades próximas sea un poco más elevado topográficamente que el resto del valle del eje fluvial Paraná-Paraguay apoyado sobre el sector chaqueño (Popolizio, 200I) pero que no limita su vulnerabilidad hídrica.

El área urbana se desarrolla sobre dos terrazas diferenciadas: una baja y pantanosa, definida como el plano de inundación y el lecho mayor del Paraná, con una extensión aproximada de Io Km, donde se localizan los puertos y las ciudades de Barranqueras y Puerto Vilelas al este de la ciudad de Resistencia y otra, más alejada y con cotas mayores, que sostiene al núcleo de Resistencia y Fontana, y centros urbanos en un radio próximo (de $15 \mathrm{Km}$ NO-NE) como Puerto Tirol, Margarita Belén y Colonia Benítez. Sobrepuesto a esta inclinación general NO-SE, el río Negro, con una serie de lagunas meándricas, y el Riacho Arazá marcan con sus recorridos otros gradientes descendentes hacia el NE y el SO.

El Gran Resistencia presenta un sistema de defensas, estaciones de bombeo y diques que regulan las crecidas ordinarias de los ríos Paraná y Paraguay resguardando gran parte de la superficie urbanizada y limitando la vulnerabilidad hídrica, pero, a pesar de este sistema de control y prevención, el sistema hidrogeomorfológico, en cierta medida, mantiene 
su dinámica o comportamiento natural. El río Negro, al igual que el río Tragadero, desborda al aumentar el caudal por el avance del Paraná sobre su cauce y conjuntamente el sistema de lagunas laterales, eleva su nivel por saturación de las napas subterráneas. Si estas situaciones ordinarias son coincidentes con precipitaciones intensas en la cuenca imbrífera del rio Negro y con repuntes o niveles altos estacionarios del eje ParanáParaguay, las áreas factibles de ser anegadas superan la cota 47 metros pudiendo llegar, en las inundaciones extraordinarias, a cota de más 50 metros; situación que advierte sobre la trascendencia de este fenómeno por cuanto la mayor parte del espacio urbano ocupado se encuentra sobre tierras factibles de ser inundadas (entre cotas de 47 y 50 metros MOP).

Como se puede inferir, el río Paraná se comporta como el gran regulador del área debido a una muy baja pendiente hidráulica entre sus afluentes, como el río Negro o el riacho Arazá. La magnitud de su valle rige, en buena medida, el comportamiento morfológico e hidrológico de sus afluentes. El drenaje de los ríos Negro y Tragadero al igual que el riacho Arazá es lento pero eficaz mientras se conserve el gradiente, pero la situación cambia cuando la elevación del nivel del agua en el Paraná lo disminuye o invierte. El aumento del nivel del río Paraná generalmente ya encuentra a los ríos Negro y Tragadero junto con el riacho Arazá crecidos y con sus valles saturados. Esta situación se acentúa cuando mayores son las alturas del río Paraná y continuas e intensas las precipitaciones en su alta cuenca.

\section{Morfología del sitio urbano del Gran Corrientes}

La ciudad de Corrientes está ubicada sobre la margen izquierda del Río Paraná, cercana a la confluencia con el Río Paraguay. El Departamento capital abarca una superficie de $522 \mathrm{~km}^{2}$ y limita al Norte y al Oeste con el Río Paraná, al Este con los Departamentos de San Cosme y San Luis y al Sur con el Departamento de Empedrado. Comprende unas 3.000 manzanas incluyendo zonas urbanas y suburbanas. Tiene una participación porcentual en el total de la población, donde el Departamento Capital ha seguido un proceso de concentración a expensas de la población provincial, con un mayor incremento que representa un $22,7 \%$ en el periodo I99I - 200I. 
$\mathrm{Si}$ se considera el aglomerado urbano que comprende el Gran Corrientes, para mitad del 2009 su población se estimaba aproximadamente en 354.000 habitantes (citypopulation, 2010), datos que la convierten en el $2^{\circ}$ aglomerado urbano del Nordeste Argentino (NEA) y el $13^{\circ}$ a nivel nacional.

Desde el punto de vista geomorfológico, la ciudad de Corrientes se encuentra inserta dentro de la denominada por el Dr. Popolizio (1977, 1997) "Gran Región Occidental de la Provincia de Corrientes"; es una gran planicie sedimentaria, comprendida entre el Río Paraná y la depresión del Iberá. Las pendientes son muy bajas, el escurrimiento es lento. Está conformado por una serie de lomadas y depresiones en las que se insertan ríos que desaguan en el Paraná. Los suelos son oscuros y muy orgánicos en las depresiones y arenosos rojizos o amarillentos, con incrustaciones calcáreas en las terrazas de los mismos.

Frente a la ciudad de Corrientes el ancho del río Paraná es de unos $4.000 \mathrm{~m}$ y la planicie de inundación tiene unos $\mathrm{I} 3 \mathrm{~km}$. El régimen del río es muy regular y presenta un marcado incremento de caudal durante los meses de verano y estiajes durante los meses invierno. En lo que hace a los cuerpos de agua que cruzan el ejido urbano, cabe mencionar los siguientes: arroyo Salamanca, Poncho Verde, Limita, Santo Domingo y Laguna Seca. Todos ellos se hallan muy antropizados, conformando actualmente parte del sistema de drenaje de la ciudad por entubamiento o escurrimiento a cielo abierto o canales.

En las últimas décadas la ciudad de Corrientes ha presentado un importante crecimiento poblacional que dio lugar a un marcado incremento en las edificaciones, lo cual ha tenido un impacto muy importante sobre el sistema de drenaje pluvial de la ciudad, afectando su funcionamiento y superando en muchos casos la capacidad de respuesta para la que fuera diseñado.

Una de las consecuencias de la convergencia de factores arriba mencionados son las inundaciones provocadas por las lluvias de cierta intensidad (por ejemplo $60 \mathrm{~mm} /$ hora) y agravadas debido a la drástica reducción de la capacidad de almacenamiento de los reservorios naturales (lagunas y superficies de absorción), por relleno de los mismos y/o por ocupación de sus márgenes; a los que se suman las inundaciones originadas por una insuficiente capacidad de los conductos de drenaje pluviales. 
Si bien ambas problemáticas tienen una duración de pocas horas, producen graves trastornos al normal funcionamiento de la ciudad. Estas inundaciones son difíciles de resolver solo con medidas estructurales (obras), por los condicionantes geomorfológicos (topografía, suelos e hidrografía) propios del emplazamiento de la ciudad, así como, por la dificultad de conseguir financiamiento para realizarlas.

Uno de los mayores problemas, posibles de superar en los sistemas de conducción pluviales urbanos, es el acarreo de basura y sedimentos en las bocas de tormenta y conductos, que obstruyen el escurrimiento provocando inundaciones.

\section{La periferia y el ambiente del Gran Resistencia y el Gran Corrientes}

En los dos primeros decenios del siglo XXI, el Gran Resistencia y el Gran Corrientes han mostrado un importante crecimiento de sus ejidos urbanos, con población que muestra diferentes caracteres socioeconómicos. En efecto, las personas que cuentan con una mejor situación económica pueden elegir el lugar donde asentarse, mientras que los grupos de clase baja y media-baja cuentan con menos oportunidades de acceso a lugares con servicios y dotados de infraestructura. De esta manera y en el siglo XXI, la creación de viviendas de tipo social por parte de los estados provinciales y municipales y localizados en zonas periféricas, ha llevado a una gran concentración de construcciones en terrenos de bajo costo y con problemas ambientales.

Por otra parte, en las últimas décadas, los terrenos vacantes comenzaron a integrarse a la trama productiva desde el punto de vista inmobiliario (Barreto y Sánchez, 2000), a pesar de que muchos de ellos están localizados en áreas ambientalmente vulnerables. Y simultáneamente, con el proceso de reurbanización sobre áreas consolidadas en las periferias del Gran Resistencia y Gran Corrientes, en las últimas décadas se produce una dispersión de actividades que conforman extensos ámbitos suburbanos de muy baja densidad sobre áreas sin urbanizar, con una marcada tendencia a la urbanización y rápidos cambios en los usos del suelo, donde se borran las improntas de las fisonomías vegetales primigenias y aparece el espacio construido, conformado espacios variables según usos y densidades de edificación (Alberto, 20II). 
Como consecuencia del proceso de avance y ocupación urbana, diferentes modificaciones del medio natural se pueden observar en la periferia de los aglomerados, pudiendo destacarse la deforestación de especies nativas, el desbroce de pastizales y la alteración de los nichos ecológicos. Asimismo, los terrenos anegables son rellenados con diferentes materiales con el objeto de favorecer la instalación humana.

Por lo tanto, la expansión urbana sin una planificación adecuada ha generado una alteración de los ambientes naturales y cambios en el propio metabolismo urbano, con grandes riesgos para la salud de la población (Organización Panamericana de la Salud, OPS, 1999). Todas estas mutaciones generan notables impactos ambientales y atentan contra los fundamentos del desarrollo urbano sostenible, ya sea por el dinamismo del fenómeno urbano, por especulaciones económicas, por políticas insuficientes o laxas (y en general) por falta de una cultura ambiental de la comunidad (Alberto, 20II; Arce et al., 20I3).

\section{El uso del suelo en la periferia del Gran Resistencia y Gran Corrientes}

Se debe considerar que existen procesos simultáneos de desarrollo urbano que se dan en la Argentina, como el de ocupación, consolidación y posterior densificación, que a su vez se enmarcan y responden a las tres lógicas del modelo de reproducción de la ciudad actual: el rol del estado o la política pública, el de los ciudadanos y sus necesidades, y el del mercado y acumulación del capital. Asimismo, estas tres lógicas se encuentran actuando en forma entremezclada, coexistiendo la ciudad del mercado, la ciudad del loteo, la ciudad de los sectores medios, la ciudad del estado a través del Fondo Nacional de la Vivienda (FONAVI), la ciudad de la necesidad con la villa y también la ciudad del mercado, con las grandes casonas de los sectores de grandes ingresos. Y todo en un ámbito urbano relativamente no muy grande (Reese, 20I2). Esta situación se reconoce en los aglomerados Gran Resistencia y Gran Corrientes, donde se reconocen esas lógicas internas y dualidades en los procesos de expansión urbana, mostrando en la periferia la existencia de los loteos particulares, la consolidación de asentamientos o barrios informales, la creación de barrios cerrados o la construcción de barrios planificados, con presencia de actores pertenecientes al estado, entidades no gubernamentales, asociaciones gremiales, propietarios privados, terciarizados o empresas inmobiliarias. 
El efecto de este fenómeno es un vaciamiento de áreas consolidadas, desarrollo de sistemas de infraestructura viaria y aumento de movilidad interurbana que acentúan el crecimiento de la mancha urbana concomitante con bolsas de marginalidad, vacíos urbanos y pobreza, a lo que se suman espacios urbanizados con un alto nivel de vulnerabilidad hídrica.

Los planes urbanos siguen siendo muy poco efectivos para regular la expansión urbana de las periferias, básicamente porque se ha considerado que las periferias urbanas son una extensión de la mancha urbana de la ciudad que tiene sus mismos componentes sociales, económicos, ambientales y urbanos. Sin embargo, investigaciones sobre periferias urbanas han demostrado que en realidad no los tienen, como tampoco responden a las mismos condicionantes legales ni de transacción inmobiliaria.

En la periferia de ambos aglomerados, predomina el uso de suelo con fin residencial, donde los grupos humanos deben recurrir a los espacios periféricos y vacíos urbanos de baja calidad ambiental que pueden encontrarse en riesgo debido a que estos territorios, lejos de reunir cualidades de habitabilidad, son frágiles y peligrosos por sus condiciones físico-naturales. La producción del suelo urbano de bajas condiciones define espacios que reúnen los componentes básicos para un desastre, tal como son el riesgo y la vulnerabilidad, que se han extendido en las últimas décadas, incrementado considerablemente las áreas urbanas de baja calidad ambiental con servicios deficientes, dando lugar a una gran mancha urbana que se caracteriza por una creciente dispersión y fragmentación territorial con un progresivo desvanecimiento de lo que se conoce como la "ciudad compacta" (Alberto, inédito:IoI).

Desde un marco de ocupación ilegal, aparecen los asentamientos informales y con la oferta legal aparecen los barrios cerrados, los complejos habitacionales y los loteos de tierras. En el cuadro comparativo (figura 6), se pueden reconocer las principales características de cada modalidad de ocupación.

Además del uso de suelo residencial, se pueden reconocer el uso de suelo comercial. Como se dijera anteriormente, exceptuando la función residencial, es importante la multiplicidad de usos del suelo en la periferia de las capitales estudiadas. No obstante, se destaca la función comercial, más aún porque las regulaciones municipales juntas con la presencia de las rutas nacionales y provinciales, atrajeron el emplazamiento de comercios que ocupan una gran superficie; donde predominan los supermercados mayoristas y minoristas, comercios vinculados 
con la construcción, la venta de maquinarias agrícolas o de automóviles.

Los clubes deportivos, la presencia de viveros e invernaderos, los centros de investigación y desarrollo (Centro Biotecnológico en Resistencia o el Centro de Ecología Aplicada del Litoral CECUAL- CONICET en Corrientes) o los cementerios-parques, integran también la periferia de estas ciudades ocupando grandes hectáreas de terrenos. Pensados para cumplir sus funciones fuera del ámbito urbano, hoy ya han sido alcanzados por el crecimiento de las ciudades.

Entonces, a las maneras tradicionales de expansión de los ejidos urbanos (barrios planificados, asentamientos informales, loteos privados, barrios privados) aparecen multitud de nuevos usos que no se pueden calificar propiamente de urbano ni exactamente de rurales tales como invernaderos y quintas semiurbanizadas, campus universitarios, almacenes mayoristas, clubes, cementerios parques, industrias aisladas, etc. (Alberto, 20I2). Este avance antrópico, sin una planificación adecuada, ha generado una alteración de los ambientes naturales y cambios en el propio metabolismo urbano, con grandes riesgos para la salud de la población (OPS, 2000). De esta manera, se manifiesta una forma de producir "fragmentos o partes de ciudad" que tienen en común el uso de la infraestructura inicial mínima e indispensable para el propio desarrollo de sus diferentes funciones, desconociendo u omitiendo cualquier interés por el territorio que, en definitiva, se irá conformando a partir de la agregación de las distintas piezas (Alcalá Pallini, 2007). 


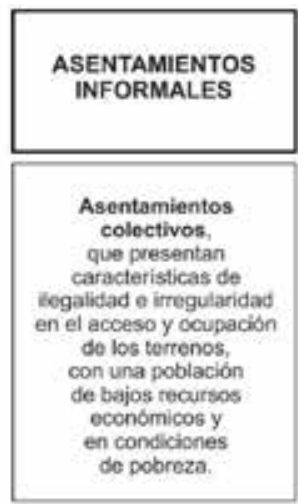

La estrategia de apropiación con grupos que tienen mayor organizacibn previa, formados con ese fin $y$ las tierras que sch ocupadas

(generaimente) son de mayor valor.

Los grupos practican diferentes formas de articulación con el poder politico y organizaciones civiles de asistencia que los han "ayudado" en la ocupación (Barreto et, al, 2000).

Situados en lugares donde no ha llegado

la infraestructura

de los servicios, en

terrenos no urbanizados

y de bajo costo

O próximos a los barrios

planificados, o sectores

con altos ingresos.

La instalación esta

tambiên en relación

con los terrenos y sus

condicionos ambientales,

además de considerar

la proximidad a las

vias de circulación.

Fuente: elaboración propia.
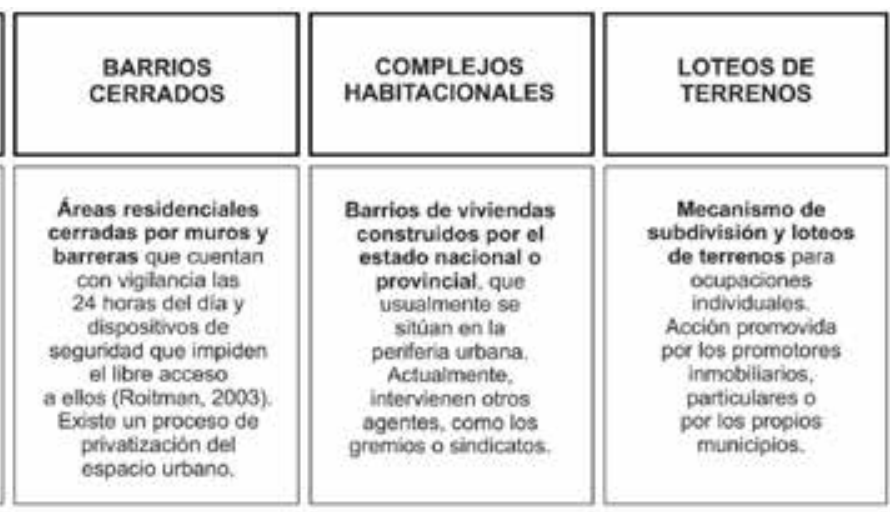

Necesided de la poblacion de clase media-alta y alta de alejarso del centro de la ciudad (ruidos molestos. contaminación visual, etc) Formas de producción que apuntan a resolver necesidades individuales o do un grupo espocifico, sin la integración de dicho coloctivo a los residentes del entorno. Fenomeno de segregación espacial; se forman

"islas de riqueza"

(Janoschika, 2002).

En las cercanias de las principales vias de circulación iruta nacionales 0 avenidas importantes de

ambos agiomerados).

Los coniuntos, se

instalan cuando el procio de la tionra es menor.

A construirse.

promueven la

instalacion de

infraestructura y

servicios bésicos y

conllevan a un aumento

en el valor del sueto

de las residencias próximas.
En la década del "60, se construyeron en forma de viviendas individuales. Descle fines de la década del 70 , se intensifican los edificios colectivos, entre 200 hasta 1000 viviendas agrupadas en pabeliones de tres o cuatro niveles.

En la decada del ' 90 ,

se vuelve al tormato de viviendas individuales. con grupos de

100 a 200 unidades

de una sola planta

Se transforman en áreas segregadas por la planificación habitacional.

Extendieron el tejdo de la ciudad vaiorizando nuevas terras urbanizables. Cracimiento en

paquetes", donde el barrio posee una comunicacion a travert de alguna via secundaria que le asegure su vinculacion a la red primaria (Alcala, 2007)

La construcción de los barrios planificados se produce mediante

"saltos" sin que exista contiguidad espacial.
Se comienza a incorporar propindades consideradas ociosas cuando la expansión do la mancha urbana estaba distante de los mismos.

Numerosas hectareas de la periferia adquieren un valor preferencial, pero se modifica la vegetacion natural del entorno. Surgidas, en primera instancia como lotes con la instalación de casas de fin de semano. actuaimente se parcela con el fin de construit barrios de viviendas.

Se reconocen los loteos en terrenos bajos y con una alta probabilidad de quedar anegadas por precipitaciones furiales. Se realizan

en la zona do transición entre los aglomerados capitales y las localidades cercanas.

Figura 6. Principales características de cada modalidad de ocupación 


\section{Los Indicadores IER (Impulso-Estado-Respuesta)}

El marco de referencia IER (Impulso - Estado - Respuesta) está basado en el concepto de la causalidad: las actividades humanas ejercen presiones sobre el ambiente, modificando la calidad y cantidad de los recursos naturales (estado). La sociedad entonces responde a estos cambios con políticas ambientales, económicas y sectoriales (Chirino et al., 2008). De esta manera, se puede incorporar en el análisis los indicadores de Impulso- Estado-Respuesta (figura 7) y en esta propuesta de estudio, se ha elaborado un esquema que permite clasificar el IER adaptando la clasificación de los indicadores ambientales propuestos para Latinoamérica según las condiciones del uso del suelo que se pueden observar en los aglomerados Gran Resistencia y Gran Corrientes (figura 8).

De esta manera, se puede reconocer que la expansión de ambos aglomerados está modificando paulatinamente la conformación del espacio periurbano y rural, y provocando diversos impactos. En primera instancia, el avance va incorporando suelos con buena aptitud agrícola donde se desarrollan diferentes cultivos (e inclusive actividades ganaderas) que generan empleo a la población local, por lo que evidentemente la disminución o desaparición de las mismas impacta en la economía local. Por otra parte, la mezcla en los usos del suelo, entre los sistemas productivos tradicionales, con los centros deportivos-recreación o con los complejos habitacionales y los barrios cerrados, pueden producir problemas de convivencia entre vecinos. Los primeros generan olores nauseabundos, animales sueltos, etc., en tanto los restantes provocan elevados niveles de ruido y movimiento de personas y vehículos, muy diferentes a los usuales en la periferia (López et al., 2015).

Con respecto a los impactos ambientales, se puede establecer que la pérdida de los espacios con cobertura vegetal profundiza los efectos de la erosión e intensifica las obstrucciones de canales y desagües, dando lugar a la acumulación de sedimentos en las depresiones y al aumento de las zonas inundadas, con pérdida de la capa fértil del suelo. La construcción de accesos, canalizaciones y nivelación para los complejos habitacionales y los barrios cerrados, está produciendo obstaculización del escurrimiento natural y modificación de niveles; la ausencia de red cloacal está provocando la contaminación de espejos de agua y napas; y los residuos domiciliarios sin un sistema eficiente de recolección y disposición final generando minibasurales (López et al., 2015). Asimismo, aparecen 
problemas de transporte y tránsito, por la sobrecarga de flujo vehicular de las avenidas de conexión con los aglomerados, que no han sido adaptadas a los requerimientos actuales, además del acceso a los barrios, ya que mayoritariamente las calles sin pavimentar no son transitadas por las líneas de transporte público en los días de lluvia.

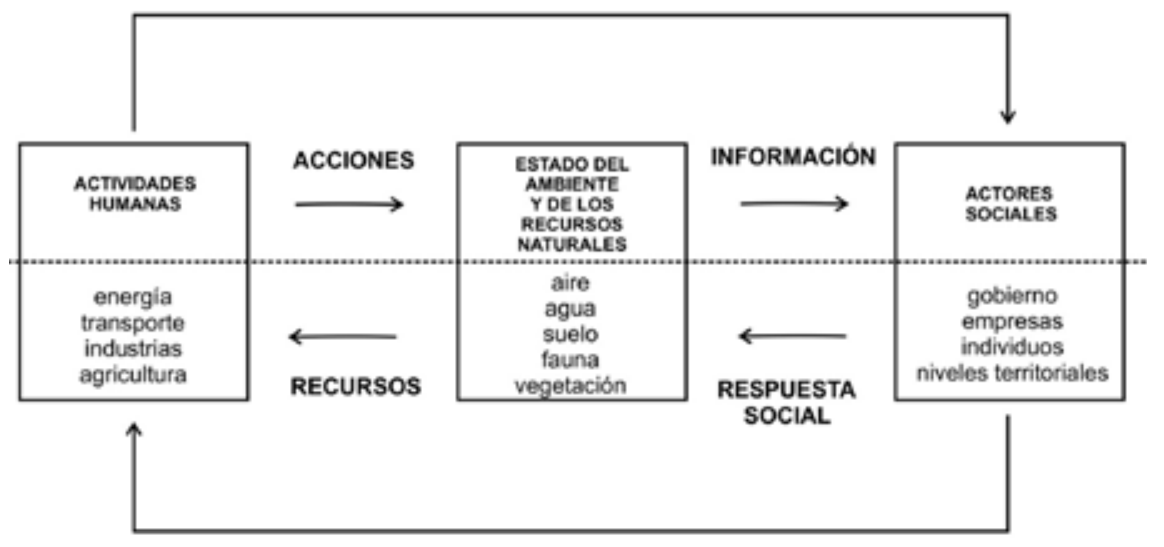

DECISIONES
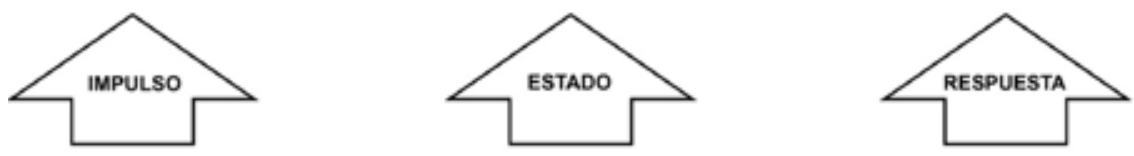

Fuente: elaborado en base a Gouzee et al., 1993; Bergar y Lams, 1996; Gupta, 2000.

Figura 7. Esquema de la organización de los indicadores de Impulso, Estado y Respuesta dónde el marco de referencia está basado en el concepto de la causalidad en relación con la dinámica ambiental

Asimismo, como se mencionara anteriormente, existen situaciones de limitación del acceso de la población a bienes públicos, ya que en las urbanizaciones cerradas quedan incluidos espejos de agua y otras áreas naturales del sistema hídrico, y además en muchos casos, la localización de estas urbanizaciones genera discontinuidad en la circulación y conexión de caminos rurales que anteriormente permitían el acceso a las mismas. Estos nuevos proyectos realizan nivelaciones y rellenos que modifican las pendientes naturales y ejecutan obras hidráulicas de desagüe, sin atención a las condiciones hidrológicas naturales de la 
cuenca (sistema lagunar y de humedales) y sin una visión de manejo integrado de la cuenca hídrica. Estas intervenciones tienen un fuerte impacto en la población más vulnerable que sufre las consecuencias de inundaciones pluviales en un sistema cada vez más antropizado y cuyo funcionamiento hidrológico ha sido alterado (López et al., 2015).

\begin{tabular}{|c|c|c|}
\hline IMPULSO & ESTADO & RESPUESTA \\
\hline $\begin{array}{l}\text { - Valor de la tierra } \\
\text { urbana y periurbana. } \\
\text { - Usos del suelo en } \\
\text { áreas restrictivas o } \\
\text { no permitidos. } \\
\text { - Avance de nuevos } \\
\text { emprendimientos } \\
\text { inmobiliarios. } \\
\text { - Loteos en áreas } \\
\text { vulnerables. } \\
\text { - Aumento de } \\
\text { la recaudación } \\
\text { fiscal municipal. } \\
\text { - Accesibilidad por } \\
\text { las vias de comunicación } \\
\text { a las Gran Resistencia } \\
\text { y Gran Corrientes. } \\
\text { - Desarrollo de planes } \\
\text { de viviendas sociales. } \\
\text { - Migraciones pendulares. } \\
\text { - Tasa de crecimiento } \\
\text { de la población urbana. }\end{array}$ & $\begin{array}{l}\text { - Parcelamiento } \\
\text { del suelo urbano } \\
\text { y periurbano. } \\
\text { - Cambio y distribución } \\
\text { en los usos del suelo. } \\
\text { - Número de loteos } \\
\text { actuales y anteriores. } \\
\text { - Ocupación de } \\
\text { terrenos fiscales. } \\
\text { - Situación dominial } \\
\text { irregular de los terrenos. } \\
\text { - Pérdida de las formaciones } \\
\text { vegetales originarias. } \\
\text { - Area urbana con servicios } \\
\text { públicos básicos. } \\
\text { - Areas cubiertas por } \\
\text { infraestructuras y } \\
\text { transporte. } \\
\text { - Espacios verdes o } \\
\text { de esparcimiento. } \\
\text { - Población en condiciones } \\
\text { de pobreza. } \\
\text { - Población en } \\
\text { barrios cerrados. }\end{array}$ & $\begin{array}{l}\text { - Actualización } \\
\text { de los Códigos de } \\
\text { Planeamiento urbano. } \\
\text { - Politicas estrategicas de } \\
\text { gestion urbana - ambiental } \\
\text { de carácter participativo. } \\
\text { - Programas de concientización } \\
\text { y educacion ambiental } \\
\text { en el ámbito formal } \\
\text { y no formal. } \\
\text { - Gestión y proteccion } \\
\text { de la biodiversidad local. } \\
\text { - Ampliacion de las } \\
\text { redes de servicios } \\
\text { públicos básicos. } \\
\text { - Rehabilitación de espacios } \\
\text { abandonados o degradados } \\
\text { por la contaminación. } \\
\text { - Organizaciones públicas y } \\
\text { privadas con programas } \\
\text { de gestión ambiental. } \\
\text { - Fortalecimiento de } \\
\text { organizaciones } \\
\text { comunitarias. }\end{array}$ \\
\hline
\end{tabular}

Figura 8. Esquema que permite clasificar el IER (ImpulsoEstado-Respuesta) adaptando la clasificación de los indicadores ambientales propuestos para Latinoamérica según las condiciones del uso del suelo que se pueden observar en los aglomerados Gran Resistencia y Gran Corrientes
Fuente: elaboración en base a OECD (1991, 1993); Chirino et al. (2008); Bataglia y Mignone (2016)

\section{Consideraciones finales}

Durante la segunda mitad del siglo XX, los aglomerados experimentaron un acelerado proceso de expansión de sus límites y la expansión de la mancha urbana fue acompañada por población de diferente condición socioeconómica mientras se iban ocupando espacios caracterizados por la falta de servicios y de infraestructura urbana. Este fenómeno no se detuvo durante los primeros ańos del siglo XXI y la ocupación de la 
periferia urbana produjo cambios en la fisonomía del paisaje natural y la aparición del paisaje construido, donde lo artificial prima a simple vista.

El avance de ambos aglomerados sobre su periferia se ha caracterizado por la ocupación y el uso de suelo residencial y comercial. Esta última función, revela numerosos locales privados mayoristas, especializados en diferentes rubros comerciales, ocupando grandes hectáreas y concentrados a la vera de las principales vías de comunicación por la necesidad de circulación de las mercancías. En cambio, los barrios cerrados y los loteos privados (inmobiliarios o individuales), más allá que se encuentran contemplados por la reglamentación municipal, también tienden a crecer por saltos y no siempre tienen en cuenta el entorno físico donde se localizan, asentándose en sectores susceptibles a las inundaciones. Además, no se produce la integración de dicho colectivo con los residentes del entorno, contribuyendo a aumentar la segregación socioespacial.

Por otra parte, la localización de los barrios planificados y las urbanizaciones cerradas, contribuyen a elevar el valor de los terrenos circundantes y dan impulso al proceso de densificación, pero esto afecta a la población de bajos recursos, que buscan terrenos más baratos sin infraestructura y por lo tanto favorecen la ocupación ilegal de terrenos y la expansión hacia las periferias más alejadas. Con respecto a esto, la presencia de los asentamientos informales con población en extrema pobreza y viviendas de baja calidad ambiental, prácticamente rodean a la periferia del Gran Corrientes y Gran Resistencia. Además, se reconoce que uno de los mayores problemas en ambas ciudades, es la falta de urbanización, ya que en ambos aglomerados la provisión de servicios, infraestructura y equipamientos es sectorizada y no alcanza a cubrir a toda la población.

El desplazamiento de población de nivel socioeconómico medio-alto al área periurbana y rural está provocando el consumo de tierras productivas que abastecen a la ciudad, y por otra parte, la subutilización de áreas urbanas tradicionalmente ocupadas por esos estratos sociales. En relación con la presunta autonomía de estos enclaves respecto de las infraestructuras y servicios de la ciudad, no es tal, en tanto se están generando diversas situaciones de impacto ambiental.

El análisis evidencia la baja integración entre lo natural y social, ya que no se tienen en cuenta los diversos tipos de actores sociales, los objetivos sectoriales, el efecto de intervenciones para obtener beneficios a corto plazo, la incidencia que esto tiene sobre los habitantes del área, la repartición de los beneficios del uso de los recursos, etc. El fenómeno aparece 
como resultado del funcionamiento del mercado inmobiliario, en el que la especulación con suelos se propaga desde el centro y los barrios de alta renta a la periferia y la rentabilidad del suelo para usos residenciales presiona sobre el uso productivo, modificando el uso de suelo a residencial. Por otra parte, el desarrollo residencial cerrado, aumentará cada vez más la superficie de espacio privatizado, haciendo cada vez más difícil el acceso y uso de bienes públicos, como espejos de agua, paisajes y otros recursos, del resto de la población.

Se debe reconocer que los municipios son los agentes estatales locales, encargados de controlar las ocupaciones y usos del suelo que surjan en la periferia urbana. Por ello se deben regular las construcciones que signifiquen restricciones al dominio en áreas urbanizables. Asimismo, es necesario actualizar los Códigos de Planeamiento urbano para que se pueda definir la intensidad y tipo de uso de suelo, además de generar acciones que regulen y controlen las diversas modalidades de ocupación, buscando evitar mayores dificultades en el futuro. En este sentido, la intervención del Estado, el accionar en la gestión del suelo y políticas urbanas está respaldado en un marco legal de regulación y control relacionados con la planificación del territorio, pero se evidencian serios inconvenientes de articulación y acción política coordinada entre los gobiernos municipales y provinciales, así como la ausencia de instrumentos que hagan operativos los procesos de intervención decidida en la regulación y el control social del suelo y la propiedad.

Esta escueta aproximación deja en claro que el crecimiento urbano implica la incorporación de nuevos territorios en el complejo sistema que comprende un espacio en mutación como lo es la ciudad, sin tener en cuenta para ello las relaciones existentes entre las demandas que incorporan estas propuestas y la dinámica de los condicionantes naturales, junto con la dinámica propia de las actividades tradicionales (agrícolas, ganaderas) previas a este proceso, lo cual complejiza las problemáticas ambientales derivadas de las transformaciones del espacio involucrado. Teniendo en cuenta los aspectos antedichos, que inciden sobre el área de estudio, surge la necesidad de profundizar e insistir en las líneas de acción futuras como:

- Estudiar las tecnologías posibles de aplicar sin pérdida de puestos de trabajo por expulsión.

- Desarrollar sistemas productivos novedosos, alternativos, complementarios y diversificados que exploten los recursos locales de manera sustentable. 
- Definir y potenciar centros críticos de desarrollo económico local, poniendo el esfuerzo en identificar núcleos urbanos que presenten un potencial desarrollo en apoyo de las actividades agropecuarias; para ello se deberá tener en cuenta:

- El papel de los actores sociales y las instituciones implicadas.

- El replanteo de políticas que obstaculizan su desarrollo y la potenciación de aquellas que lo favorecen.

- Definir cuáles son los estrangulamientos que impiden un funcionamiento más eficaz.

- Trabajar en el incremento del intercambio comercial entre centros poblados cercanos para que se conviertan, además, en centros de distribución de los productos agrarios, fuentes para abastecer de conocimientos técnicos y recursos para la elaboración de productos destinados al consumo provincial.

- Paliar el acceso insuficiente o la mala distribución de tierras-ya sea por obstáculos políticos, sociales, inmobiliarios o económicos-, una de las barreras más graves para el desarrollo sustentable, ya sea que tengan fines productivos o residenciales.

- Legislar para limitar la usurpación de tierras por medio de un sistema no sujeto a la escrituración usual de la propiedad privada, para ello se deberá determinar la propiedad de las parcelas y registrar el derecho de los individuos sobre los terrenos, a partir de normas que resulten accesibles a los grupos desfavorecidos y de bajos ingresos.

- Proponer medidas enérgicas para limitar la expansión urbana en las zonas agrícolas fértiles, conservándolas para la producción de alimentos y como espacios libres de contaminación o corredores verdes.

- Construir la estructura que oriente el cambio de la actividad urbana y de la actividad rural, trabajando a favor de educar en una cultura acorde con el desarrollo sustentable.

Finalmente, se considera que la administración, planificación y ordenación de un territorio no es feudo de nadie, o mejor dicho, nadie es dueño de la verdad por más modelo de proyección que se plantee. La administración y organización del territorio son, en gran parte, productos que reflejan los valores y supuestos de una cultura determinada. 


\section{Referencias bibliográficas}

Alberto, J. (20II). Las fronteras urbanas. Escenarios de transición, vulnerabilidad y conflictos en el Área Metropolitana del Gran Resistencia, en A. M. Foschiatti (coord.). Escenarios Vulnerables del Nordeste Argentino (pp. 64-95). Corrientes, Argentina: UNNE, ANPCyT y Conicet.

Alberto, J. A. (2012). Papel de los geoindicadores en el análisis espacial. Una herramienta de apoyo a la ciencia geográfica, Revista Geográfica Digital, Vol. 9 (I7), pp. I-I5.

Alcalá Pallini, L. (2007). Dimensiones urbanas del problema habitacional. El caso de la ciudad de Resistencia, Argentina, Revista INVI, Vol. 22 (59), pp. 35- 68.

Arce, G.; López, S. y Alberto, J. (2013). Propuesta de geoindicadores en desarrollo sostenible en espacios periurbanos. Estudio de caso en la ciudad de Corrientes, en Anales del XXXIII Encuentro de Geohistoria Regional [CDRom], Formosa, Formosa: Universidad Nacional de Formosa.

Barreto, M. y Sánchez, L. (200o). El crecimiento de las ciudades intermedias del noreste argentino en el contexto de las transformaciones regionales, en Seminario El rol de las ciudades intermedias iberoamericanas [CD-Rom], Resistencia, Chaco: Facultad de Arquitectura y Urbanismo, UNNE. [Versión en CD-Rom]

Bataglia, M. A. (septiembre, 2012). Aplicación y alcances en el aula de geoindicadores. Panel: Los geoindicadores como herramientas geográficas para el análisis espacial y su aplicación en el aula, en Actas del XIV Encuentro de Profesores de Geografía del Nordeste: "Nuevos Espacios, problemas y desafios para la Geografía del Siglo XXI' [CD-Rom], Resistencia, Chaco: Facultad de Humanidades, UNNE.

Bataglia, M. y Mignone, A. (20r6). La localidad de Puerto Tirol (Chaco) en el siglo XXI. Un diagnóstico mediante la aplicación de Geoindicadores, en XIV Encuentro de Profesores y Licenciados de Geografía de Formosa [CD-Rom], Formosa, Formosa: Universidad Nacional de Formosa.

Berger, A. e Iams, W.J. (I996). Geoindicators: Assessing rapid environmental changes in Earth systems. Rotterdam, A. Balkema.

Bonetto, A. y Orfeo, O. (1984). Caracteres sedimentológicos de la carga en suspensión del río Paraná entre Confluencia y Esquina (prov. De Corrientes, R.A.), Asociación Argentina de Mineralogía, Petrología y Sedimentología, T. Is (3-4), Julio-Diciembre, Bs As. Argentina. 
Capel, H. (1994). Las periferias urbanas y la geografía. Reflexiones para arquitectos, en CAPEL H. La geografía hoy. Textos, historia y documentación, Materiales de trabajo intelectual, Barcelona: Anthropos, 1994, No 43.

Citypopulation (2010). Argentina: Aglomeraciones, Ciudades y Localidades [En línea] Recuperado de: https:/www.citypopulation.de/Argentina-Cities.html

Chirino, E.; Abad, J. y Bellot, J. (2008). Uso de indicadores de PresiónEstado-R en el diagnóstico de la comarca de la Marina Baixa, SE, España, en Indicadores y tecnologias apropiadas para el uso del agua en las Tierras Secas de Iberoamérica. El agua en Iberoamérica. XIII, Mendoza: CYTED [En línea] Recuperado de: http://www.cricyt.edu.ar/ladyot/publicaciones/cyted libro XIII/pdf/2 chirino.pdf

Coltrinari, L. (200I). Geoindicadores de cambios globales rápidos en los trópicos húmedos, en VIII Encuentro de Geógrafos de América Latina. Santiago (Chile): Universidad de Chile [En línea] Recuperado de: http:// geodados.pg.utfpr.edu.br/busca/detalhe.php?id=23I45

Gallopín, G.C. (2006). Sostenibilidad y desarrollo sostenible: un enfoque sistémico, Serie Medio Ambiente y Desarrollo, No 64, División de Desarrollo Sostenible y Asentamientos Humanos, Santiago, Chile: CEPAL,

Google Earth. (20I7). Imagen satelital del Gran Resistencia y Gran Corrientes.

Gouzee, N.; Mazijn, B. y Billharz, S. (1993). Indicators of Sustainable Development for Decision - Making. 5-8.

Gupta, A. (agosto, 2000). Geoindicators for tropical urbanization. Conferencia realizada en el Simposio Geoindicators: Applications to the Humid Tropics [Manuscrito] Rio de Janeiro, Brasil. 3Ist International Geological Congress.

Gutiérrez Puebla, J. (1984). La ciudad y la organización regional, Cuadernos de Estudios, NoI4. Serie Geografía. Madrid, España: Cincel.

IUGS. (2012). Introducción a los geoindicadores, en IUGS Geoindicators Initiative (GEOIN) [En línea] Recuperado de: http://www.lgt.lt/geoin/files/ spanish flyer.pdf.

Janoschka, M. (2002). El nuevo modelo de la ciudad latinoamericana: fragmentación y privatización, Revista EURE, Vol.28, $\mathrm{N}^{\circ} 85$.

López, S.; Arce, G. y Alberto, J. (2015). Geoindicadores aplicados al estudio de impactos ambientales en el área periurbana de la ciudad de Corrientes, Revista Geográfica Digital, Vol. I2 (23), Resistencia, Chaco: IGUNNE, Facultad de Humanidades, UNNE [En línea] Recuperado de: http://hum. unne.edu.ar/revistas/geoweb/Geo23/archivos/cga23.pdf 
Neiff, J., Poi de Neiff, A. y Casco, S. (2005). Importancia ecológica del Corredor Fluvial Paraguay-Paraná como contexto del manejo sostenible, en Peteán, J. y J. Cappato, Humedales fluviales de América del Sur. Hacia un manejo sustentable, Fundación Proteger.

OCDE. (1993). OECD Core Set of Indicators for Environmental Performance Reviews, Environmental, Monograph, N 83, Paris, Francia. OCDE.

OCDE. (I99I). Environmental Indicators: A Preliminary Set. París, Francia. OCDE.

OPS. Organización Panamericana de la Salud (2000). Políticas de salud en la vivienda [En línea] Recuperado de: www.cepis.ops-oms.org

OPS. Organización Panamericana de la Salud (1999). Documentos OPS sobre politica de salud en la vivienda. Washington, D.C. y La Habana, Cuba: División de Salud y Ambiente de la Organización Panamericana de la Salud.

Orfeo, O. (1997). Comparación sedimentológica y geomorfológica de los ríos Paraná y Paraguay en el área de su confluencia, Memorias del I Congreso Latinoamericano de Sedimentología. Sociedad Venezolana de Sedimentología. Soc. Venezolana de Geología, Tomo II I29-I33 Porlamar, Isla Margarita, Venezuela.

Orfeo, O. (1996). Geomorfología del sistema fluvial Paraná-Paraguay en el área de su confluencia, XIII Congreso Geológico Argentino y III Congreso de exploración de hidrocarburos. Actas IV I3I-I47. Bs As. Argentina. Octubre de 1996.

Popolizio, E. (200I). Los cambios de posición del valle del Río Paraná a lo largo de su historia geomorfológica. Comunicaciones Científicas y Tecnológicas, Secretaría General de Ciencia y Técnica. UNNE, Resistencia. Chaco [En línea] Recuperado de: http://www.unne.edu.ar/unnevieja/Web/cyt/ cyt/200I/7-Tecnologicas/T-082.pdf

Popolizio, E. (200o). El Paraná, un río y su historia geomorfológica.

Comunicaciones Científicas y Tecnológicas, Secretaría General de Ciencia y Técnica. UNNE. Resistencia. Chaco [En línea] Recuperado de: http://www. unne.edu.ar/unnevieja/Web/cyt/cyt/200o/7-Tecnologicas/T-034.pdf

Popolizio, E. (1975). Contribución a la Geomorfología de la Provincia de Corrientes, Serie A - Notas No 8 y 9. Instituto de Fisiografía y Geología - Facultad de Ciencias Exactas e Ingeniería, Universidad Nacional de Rosario.

Popolizio, E. (I966). Causas geográficas de los desplomes y deslizamientos de las riberas del Río Paraná y en especial en la ciudad de Corrientes”, Rev. Univ. Lambda, No 6-7. 
Reese, E. (2012). Instrumentos de Gestión urbana fortalecimiento del rol del municipio y desarrollo con equidad. Buenos Aires, Argentina: Instituto del Conurbano de la Universidad Nacional de General Sarmiento.

Roitman, S. (2003). Barrios cerrados y segregación social urbana, Scripta Nova: Revista de Geografia y Ciencias Sociales, Vol. VII, N ${ }^{\circ} 46$ (II8).

Vapnarsky, C. A. y Gorojovsky, N. (1990). El crecimiento urbano en la Argentina. Buenos Aires: Grupo Editor Latinoamericano. IIED. América Latina. 